\title{
Complexity of determining the most vital elements for the 1-median and 1-center location problems
}

\author{
Cristina Bazgan, Sonia Toubaline, and Daniel Vanderpooten \\ Université Paris-Dauphine, LAMSADE, \\ Place du Maréchal de Lattre de Tassigny, 75775 Paris Cedex 16, France. \\ \{bazgan, toubaline, vdp\}@lamsade.dauphine.fr
}

\begin{abstract}
We consider the $k$ most vital edges (nodes) and min edge (node) blocker versions of the 1-median and 1-center location problems. Given a weighted connected graph with distances on edges and weights on nodes, the $k$ most vital edges (nodes) 1-median (respectively 1-center) problem consists of finding a subset of $k$ edges (nodes) whose removal from the graph leads to an optimal solution for the 1-median (respectively 1-center) problem with the largest total weighted distance (respectively maximum weighted distance). The complementary problem, min edge (node) blocker 1-median (respectively 1-center), consists of removing a subset of edges (nodes) of minimum cardinality such that an optimal solution for the 1-median (respectively 1-center) problem has a total weighted distance (respectively a maximum weighted distance) at least as large as a specified threshold. We show that $k$ most vital edges 1median and $k$ most vital edges 1-center are $N P$-hard to approximate within a factor $\frac{7}{5}-\epsilon$ and $\frac{4}{3}-\epsilon$ respectively, for any $\epsilon>0$, while $k$ most vital nodes 1 -median and $k$ most vital nodes 1 -center are $N P$-hard to approximate within a factor $\frac{3}{2}-\epsilon$, for any $\epsilon>0$. We also show that the complementary versions of these four problems are $N P$-hard to approximate within a factor 1.36 .
\end{abstract}

Keywords: most vital edges and nodes, 1-median, 1-center, complexity, approximation.

\section{Introduction}

For problems of security or reliability, it is important to assess the ability of a system to resist to a destruction or a failure of a number of its entities. This amounts to identifying critical entities which can be determined with respect to a measure of performance or a cost associated to the system. In this paper we focus on simple location problems. Consider for instance the following problem. We aim at locating one hospital or one supermarket in order to serve $n$ areas. Each area is characterized by a population which represents a potential demand. 
The areas are connected by roads with a given distance. The objective for locating this hospital or supermarket is not the same. Indeed, for the hospital, we aim at finding the location that minimizes the maximum distance weighted by population from the hospital to all areas while for the supermarket we aim at finding the location that minimizes the total weighted distance from the supermarket to all areas. However, there may occur incidents such as works on road or floods that make some roads inaccessible. In this case several problems may arise. We can aim at detecting the critical roads whose failure causes the largest increase in the weighted distance. Alternatively, wa can aim at determining the maximum number of damaged roads which still ensures a certain quality of service level. Modeling the considered network by a weighted connected graph with distances on edges and weights on nodes, where roads are edges and areas are nodes, these problems consist either of finding among all subset of edges or nodes, a subset whose removal from the graph generates the largest increase in the total or maximum weighted distance or of determining a subset of edges or nodes of minimal cardinality such that, when we remove this subset from the graph, the total or maximum weighted distance is at least as large as a specified threshold. In the literature these problems are referred respectively to as the $k$ most vital edges/nodes and the min edge/node blocker problems.

The $k$ most vital edges/nodes and min edge/node blocker versions have been studied for several problems, including shortest path, minimum spanning tree, maximum flow, maximum matching and independent set. The $k$ most vital edges problem with respect to shortest path was proved $N P$-hard [2]. Later, $k$ most vital edges/nodes shortest path (and min edge/node blocker shortest path, respectively) were proved not 2-approximable (not 1.36-approximable, respectively) if $P \neq N P$ [8]. For minimum spanning tree, $k$ most vital edges is NP-hard [6] and $O(\log k)$-approximable [6]. In [11] it is proved that $k$ most vital edges maximum flow is $N P$-hard. For maximum matching, min edge blocker is $N P$-hard even for bipartite graphs [12], but polynomial for grids and trees [10]. In [3], the $k$ most vital nodes and min node blocker versions with respect to independent set for bipartite graphs remain polynomial on the unweighted graphs and become NP-hard for weighted graphs. For bounded treewidth graphs and cographs these versions remain polynomial [3]. Concerning the approximation on bipartite weighted graphs, $k$ most vital nodes with respect to independent set has no ptas [3].

In this paper the $k$ most vital edges (nodes) and min edge (node) blocker versions for the 1-median and 1-center problems are studied.

After introducing some preliminaries in Section 2, we prove in Section 3 that $k$ Most Vital Edges (Nodes) 1-Median (1-Center) and Min Edge (NODE) BLOCKER 1-MEDIAN (1-CENTER) are not constant approximable for some constants, unless $P=N P$. Final remarks are provided in Section 4. 


\section{Basic concepts and definitions}

Consider $G=(V, E)$ a connected weighted graph with $|V|=n$ and $|E|=m$. Let $d_{v_{i} v_{j}}$ be the distance between $v_{i}$ and $v_{j}$ for $\left(v_{i}, v_{j}\right) \in E$ and $w_{v_{i}}$ be the weight associated to node $v_{i}$ for $i=1, \ldots, n$ ( $w_{v_{i}}$ represents the demand occurring at node $\left.v_{i}\right)$. Denote by $d\left(v_{i}, v_{j}\right)$ the minimum distance between two nodes $v_{i}$ and $v_{j}$ of $G$. The 1-median (respectively 1-center) problem consists of locating the median (respectively the center) of a graph $G$, that is the node $v$ which minimizes the total weighted distance (respectively the maximum weighted distance) to all nodes of the graph given by $\sum_{v_{i} \in V} w_{v_{i}} d\left(v, v_{i}\right)$ (respectively $\max _{v_{i} \in V} w_{v_{i}} d\left(v, v_{i}\right)$ ).

Denote by $G-R$ the graph obtained from $G$ by removing the subset $R$ of edges or nodes.

We consider in this paper the $k$ most vital edges (nodes) and min edge (node) blocker versions of the 1-median and 1-center problems. These problems are defined as follows:

\section{$k$ Most Vital Edges 1-median (1-Center)}

Input: A connected graph $G=(V, E)$ weighted by two functions $d: E \rightarrow N$ and $w: V \rightarrow N$ and a positive integer $k$.

Output: A subset $S^{*} \subseteq E$, with $\left|S^{*}\right|=k$, whose removal generates an optimal solution for the 1-median (1-center) problem in the graph $G-S^{*}$ of maximal value.

\section{$k$ Most Vital Nodes 1-median (1-Center)}

Input: A connected graph $G=(V, E)$ weighted by two functions $d: E \rightarrow N$ and $w: V \rightarrow N$ and a positive integer $k$.

Output: A subset $N^{*} \subseteq V$, with $\left|N^{*}\right|=k$, whose removal generates an optimal solution for the 1-median (1-center) problem in the graph $G-N^{*}$ of maximal value.

Min EdGe BLOCKer 1-Median (1-CENTER)

Input: A connected graph $G=(V, E)$ weighted by two functions $d: E \rightarrow N$ and $w: V \rightarrow N$ and a positive integer $U$.

Output: An edge blocker $S^{*} \subseteq E$ of minimal cardinality where an edge blocker is a subset of edges such that the value of an optimal solution for the 1-median (1-center) problem in the graph $G-S^{*}$ is greater than or equal to $U$.

Min Node Blocker 1-Median (1-CENTER)

Input: A connected graph $G=(V, E)$ weighted by two functions $d: E \rightarrow N$ and $w: V \rightarrow N$ and a positive integer $U$.

Output: A node blocker $N^{*} \subseteq V$ of minimal cardinality where a node blocker is a subset of nodes such that the value of an optimal solution for the 1-median (1-center) problem in the graph $G-N^{*}$ is greater than or equal to $U$.

Given an NPO optimization problem and an instance $I$ of this problem, we use $|I|$ to denote the size of $I, \operatorname{opt}(I)$ to denote the optimum value of $I$, and 
$\operatorname{val}(I, S)$ to denote the value of a feasible solution $S$ of instance $I$. The performance ratio of $S$ (or approximation factor) is $r(I, S)=\max \left\{\frac{\operatorname{val}(I, S)}{\operatorname{opt}(I)}, \frac{\operatorname{opt}(I)}{\operatorname{val}(I, S)}\right\}$. The error of $S, \varepsilon(I, S)$, is defined by $\varepsilon(I, S)=r(I, S)-1$.

For a function $f$, an algorithm is an $f(n)$-approximation, if for every instance $I$ of the problem, it returns a solution $S$ such that $r(I, S) \leq f(|I|)$.

The notion of a gap-reduction was introduced in [1] by Arora and Lund. A minimization problem $\Pi$ is called gap-reducible to a maximization problem $\Pi^{\prime}$ with parameters $(c, \rho)$ and $\left(c^{\prime}, \rho^{\prime}\right)$, if there exists a polynomial time computable function $f$ such that $f$ maps an instance $I$ of $\Pi$ to an instance $I^{\prime}$ of $\Pi^{\prime}$, while satisfying the following properties.

- If $\operatorname{opt}(I) \leq c$ then $\operatorname{opt}\left(I^{\prime}\right) \geq c^{\prime}$

- If $\operatorname{opt}(I)>c \rho$ then $\operatorname{opt}\left(I^{\prime}\right)<\frac{c^{\prime}}{\rho^{\prime}}$

Parameters $c$ and $\rho$ are function of $|I|$ and parameters $c^{\prime}$ and $\rho^{\prime}$ are function of $\left|I^{\prime}\right|$. Also, $\rho, \rho^{\prime} \geq 1$.

The interest of a gap-reduction is that if $\Pi$ is not approximable within a factor $\rho$ then $\Pi^{\prime}$ is not approximable within a factor $\rho^{\prime}$.

The notion of an $E$-reduction (error-preserving reduction) was introduced in [9] by Khanna et al. A problem $\Pi$ is called E-reducible to a problem $\Pi^{\prime}$, if there exist polynomial time computable functions $f, g$ and a constant $\beta$ such that

- $f$ maps an instance $I$ of $\Pi$ to an instance $I^{\prime}$ of $\Pi^{\prime}$ such that $\operatorname{opt}(I)$ and $\operatorname{opt}\left(I^{\prime}\right)$ are related by a polynomial factor, i.e. there exists a polynomial $p$ such that $\operatorname{opt}\left(I^{\prime}\right) \leq p(|I|) \operatorname{opt}(I)$,

- $g$ maps any solution $S^{\prime}$ of $I^{\prime}$ to one solution $S$ of $I$ such that $\varepsilon(I, S) \leq$ $\beta \varepsilon\left(I^{\prime}, S^{\prime}\right)$.

An important property of an $E$-reduction is that it can be applied uniformly to all levels of approximability; that is, if $\Pi$ is $E$-reducible to $\Pi^{\prime}$ and $\Pi^{\prime}$ belongs to $\mathcal{C}$ then $\Pi$ belongs to $\mathcal{C}$ as well, where $\mathcal{C}$ is a class of optimization problems with any kind of approximation guarantee (see also [9]).

\section{$3 \quad N P$-hardness of approximation}

We first prove that $k$ Most Vital Edges (Nodes) 1-median and $k$ Most Vital Edges (Nodes) 1-CENTER are not constant approximable for some constants, unless $P=N P$. For this, we construct, in theorems 1 and 2, gap-reductions from Min VerTex COVER restricted to tripartite graphs. This problem is shown $N P$-hard in [7] where Garey et al. prove that it is $N P$-hard to find a minimum vertex cover in graphs of maximum degree 3 , considering also that these graphs, with the exception of the clique $K_{4}$, are 3-colorable [4]. 
Theorem 1. $k$ Most Vital Edges 1-Median and $k$ Most Vital Edges 1CENTER are NP-hard to approximate within a factor $\frac{7}{5}-\epsilon$ and $\frac{4}{3}-\epsilon$ respectively, for any $\epsilon>0$.

Proof: We first consider $k$ Most Vital Edges 1-Median.

Let $I$ be an instance of Min VerTex Cover formed by a graph $G=(V, E)$ with a tripartition $V=V_{1} \cup V_{2} \cup V_{3}$ and $|V|=n$. We construct an instance $I^{\prime}$ of $k$ Most Vital Edges 1-Median consisting of a graph $G^{\prime}=\left(V^{\prime}, E^{\prime}\right)$ with $k<n$ as follows (see Figure 1). We associate for each node $v_{\ell}^{i} \in V_{i}$, two nodes $v_{\ell, 1}^{i}$ and $v_{\ell, 2}^{i}$ in $V^{\prime}$ and connect them in $E^{\prime}$, for $i=1,2,3$ and $\ell=1, \ldots,\left|V_{i}\right|$. We add for each edge $\left(v_{\ell}^{i}, v_{r}^{j}\right) \in E$, with $i<j$, the edge $\left(v_{\ell, 2}^{i}, v_{r, 1}^{j}\right)$ to $E^{\prime}$. We also add four nodes $x_{1}, x_{2}, x_{2}^{\prime}, x_{3}$ connected by the path $\left(x_{1}, x_{2}^{\prime}\right),\left(x_{2}^{\prime}, x_{2}\right),\left(x_{2}, x_{3}\right)$. We connect $x_{1}$ to $v_{\ell, 1}^{1}$ for $\ell=1, \ldots,\left|V_{1}\right|, x_{2}^{\prime}$ to $v_{\ell, 1}^{2}$ and $x_{2}$ to $v_{\ell, 2}^{2}$ for $\ell=1, \ldots,\left|V_{2}\right|$ and $x_{3}$ to $v_{\ell, 2}^{3}$ for $\ell=1, \ldots,\left|V_{3}\right|$. We assign a distance 1 to edges $\left(x_{1}, x_{2}^{\prime}\right)$, $\left(x_{1}, v_{\ell, 1}^{1}\right),\left(x_{2}^{\prime}, v_{j, 1}^{2}\right),\left(x_{2}, v_{j, 2}^{2}\right)$ and $\left(x_{2}^{\prime}, x_{3}\right)$ for $\ell=1, \ldots,\left|V_{1}\right|$ and $j=1, \ldots,\left|V_{2}\right|$, a distance 2 for the edge $\left(x_{2}^{\prime}, x_{2}\right)$ and a distance 0 for all the other edges in $E^{\prime}$. We set $w_{x_{1}}=8, w_{x_{2}}=w_{x_{3}}=1$ and assign a weight 0 to all other nodes in $V^{\prime}$. We replace all edges of $E^{\prime}$, except the edges $\left(v_{\ell, 1}^{i}, v_{\ell, 2}^{i}\right)$, for $i=1,2,3$ and $\ell=1, \ldots,\left|V_{i}\right|$, by the gadget given in Figure 2. For each edge to be replaced, one chooses indifferently the vertex playing the role of $i$ in Figure 2, except for all edges incident to $x_{1}$ for which we take $x_{1}$ as $i$. We show in the following that:

1. $\operatorname{opt}(I) \leq k \Rightarrow \operatorname{opt}\left(I^{\prime}\right) \geq 7$

2. $\operatorname{opt}(I)>k \Rightarrow \operatorname{opt}\left(I^{\prime}\right) \leq 5$

which proves that $k$ Most Vital Edges 1-MEdian is $N P$-hard to approximate within a factor $\frac{7}{5}-\epsilon$, for any $\epsilon>0$.

First observe that there exists at least one optimal solution of $k$ MOST VITAL EDGES 1-MEDIAN containing only edges among the edges $\left(v_{\ell, 1}^{i}, v_{\ell, 2}^{i}\right)$, for $i=$ $1,2,3$ and $\ell=1, \ldots,\left|V_{i}\right|$. Indeed, if a solution contains edges from a gadget corresponding to an initial edge $(i, j)$, it must contain at least $n$ edges from this gadget in order to have a chance to increase the solution value by suppressing communication between $i$ and $j$. Therefore, since $k<n$, it is at least as good to select $k$ edges among those which do not belong to the gadgets.

Observe also that $G^{\prime}$ is designed so as to ensure that $x_{1}$ will always be the optimal 1-median node. Indeed, since the weight of vertex $x_{1}$ is 8 and all edges incident to $x_{1}$ have distance 1 , any other node would have a total weighted distance of at least 8 . In the following, $x_{1}$ has always a total distance of at most 7 .

1. If there exists a vertex cover $V^{\prime} \subseteq V$ of cardinality less than $k$ in $G$ then consider any set of vertices $V^{\prime \prime} \supset V^{\prime}$ of cardinality $k$, and remove $S^{\prime \prime}=$ $\left\{\left(v_{\ell, 1}^{i}, v_{\ell, 2}^{i}\right): v_{\ell}^{i} \in V^{\prime \prime}\right\}$ from $G^{\prime}$. The optimal 1-median node in $G^{\prime}-S^{\prime \prime}$ is $x_{1}$ with a total weighted distance $d\left(x_{1}, x_{2}\right)+d\left(x_{1}, x_{3}\right)=3+4=7$. Hence, $\operatorname{opt}\left(I^{\prime}\right) \geq 7$. 


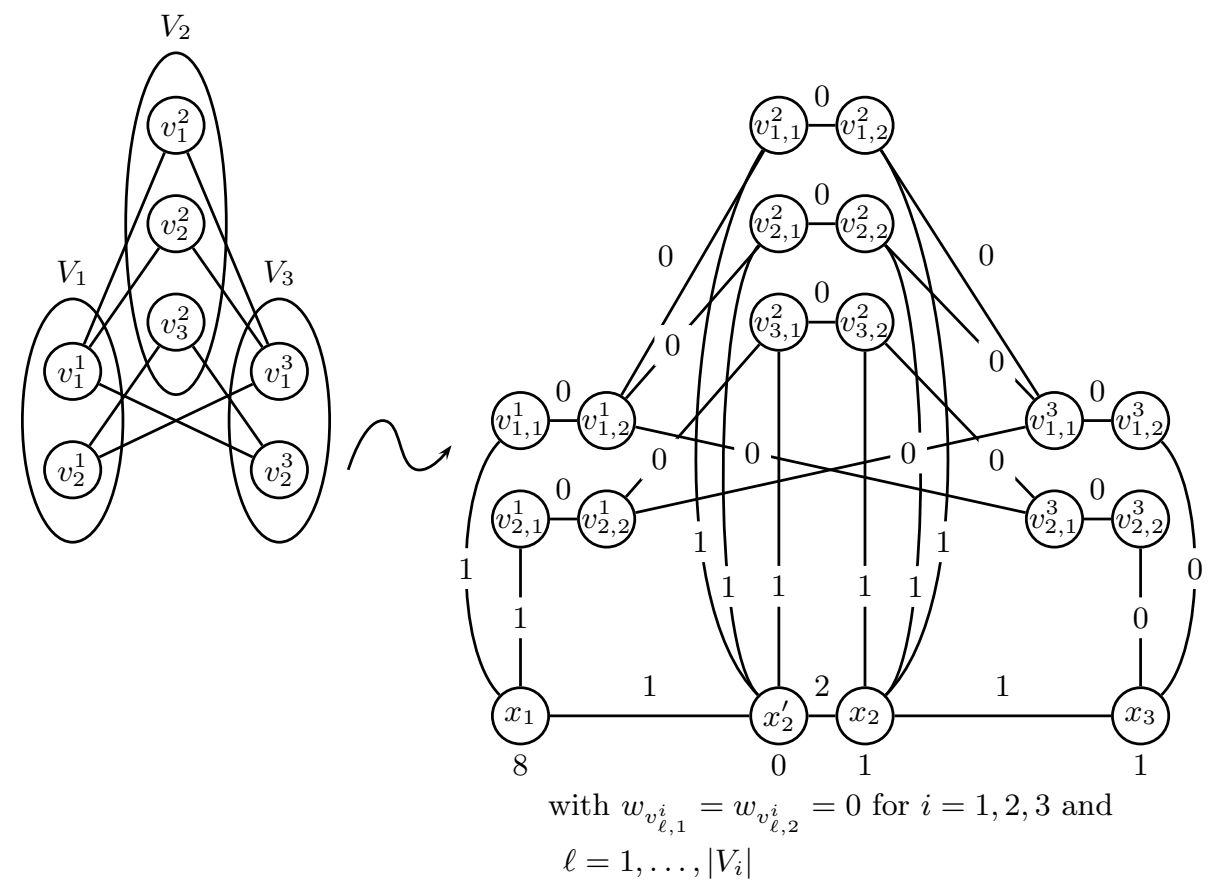

Fig. 1. Construction of $G^{\prime}$ from $G$
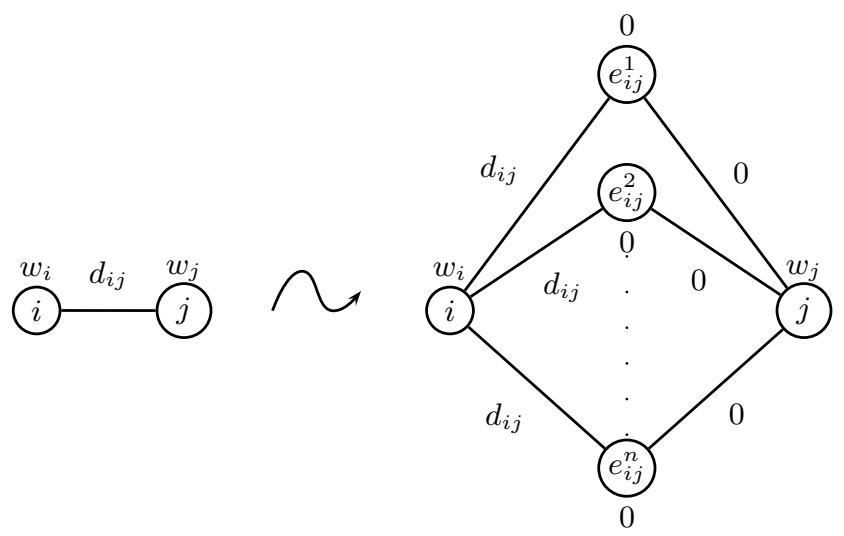

Fig. 2. The replacement gadget of an edge $e=(i, j) \in E^{\prime}$ 
2. Let $S^{*}$ be any solution of $k$ Most Vital Edges 1-MEdian which contains only edges $\left(v_{\ell, 1}^{i}, v_{\ell, 2}^{i}\right)$, for $i=1,2,3$ and $\ell=1, \ldots,\left|V_{i}\right|$. The optimal 1median node in $G^{\prime}-S^{*}$ is $x_{1}$ with $\operatorname{opt}\left(I^{\prime}\right)=d\left(x_{1}, x_{2}\right)+d\left(x_{1}, x_{3}\right)$. Each edge $\left(v_{\ell, 1}^{i}, v_{\ell, 2}^{i}\right)$ of $S^{*}$ corresponds to a node $v_{\ell}^{i} \in V_{i}$ in the graph $G$, for $i=1,2,3$ and $\ell=1, \ldots,\left|V_{i}\right|$. Let $N^{*}$ be the subset of nodes in $G$ that correspond to edges of $S^{*}$. Since $\left|N^{*}\right|=k$ and $\operatorname{opt}(I)>k, N^{*}$ is not a vertex cover in $G$. Thus, there exists at least one edge $\left(v_{\ell}^{i}, v_{r}^{j}\right) \in E$ which is not covered. This implies in $G^{\prime}$ the existence of a path from $x_{i}$ (or $x_{i}^{\prime}$ ) to $x_{j}$, with $i<j$, passing through the gadget corresponding to the edge $\left(v_{\ell, 2}^{i}, v_{r, 1}^{j}\right)$, enabling a decrease of some shortest path distances. Hence,

- if $i=1$ and $j=2$ then $\operatorname{opt}\left(I^{\prime}\right) \leq 6$

- if $i=1$ and $j=3$ then $\operatorname{opt}\left(I^{\prime}\right) \leq 3$

- if $i=2$ and $j=3$ then $\operatorname{opt}\left(I^{\prime}\right) \leq 6$

Therefore, $\operatorname{opt}\left(I^{\prime}\right) \leq 6$.

We consider now $k$ Most Vital Edges 1-Center. We use the same construction as above. We show that:

1. $\operatorname{opt}(I) \leq k \Rightarrow \operatorname{opt}\left(I^{\prime}\right) \geq 4$

2. $\operatorname{opt}(I)>k \Rightarrow \operatorname{opt}\left(I^{\prime}\right) \leq 3$

which proves that $k$ Most Vital Edges 1-CEnTER is NP-hard to approximate within a factor $\frac{4}{3}-\epsilon$, for any $\epsilon>0$.

Similarly as above, there exists at least one optimal solution of $k$ MosT VITAL EDGES 1-CENTER containing only edges among the edges $\left(v_{\ell, 1}^{i}, v_{\ell, 2}^{i}\right)$, for $i=1,2,3$ and $\ell=1, \ldots,\left|V_{i}\right|$. Moreover, as before, $x_{1}$ will always be the optimal 1 -center node.

1. If there exists a vertex cover $V^{\prime} \subseteq V$ of cardinality less than $k$ in $G$ then consider any set of vertices $V^{\prime \prime} \supset V^{\prime}$ of cardinality $k$, and remove $S^{\prime \prime}=$ $\left\{\left(v_{\ell, 1}^{i}, v_{\ell, 2}^{i}\right): v_{\ell}^{i} \in V^{\prime \prime}\right\}$ from $G^{\prime}$. The optimal 1-center node in $G^{\prime}-S^{\prime \prime}$ is $x_{1}$ with a maximum weighted distance $\max \left\{d\left(x_{1}, x_{2}\right), d\left(x_{1}, x_{3}\right)\right\}=4$. Hence, $\operatorname{opt}\left(I^{\prime}\right) \geq 4$.

2. Let $S^{*}$ be any solution of $k$ MOst Vital EdGes 1-CENTER which contains only edges $\left(v_{\ell, 1}^{i}, v_{\ell, 2}^{i}\right)$, for $i=1,2,3$ and $\ell=1, \ldots,\left|V_{i}\right|$. The optimal 1-center node in $G^{\prime}-S^{*}$ is $x_{1}$ with $\operatorname{opt}\left(I^{\prime}\right)=\max \left\{d\left(x_{1}, x_{2}\right), d\left(x_{1}, x_{3}\right)\right\}$. Each edge $\left(v_{\ell, 1}^{i}, v_{\ell, 2}^{i}\right)$ of $S^{*}$ corresponds to a node $v_{\ell}^{i} \in V_{i}$ in the graph $G$, for $i=1,2,3$ and $\ell=1, \ldots,\left|V_{i}\right|$. Let $N^{*}$ be the subset of nodes of $G$ corresponding to edges in $S^{*}$. Since $\left|N^{*}\right|=k$ and $\operatorname{opt}(I)>k, N^{*}$ is not a vertex cover in $G$. Thus, there exists at least one edge $\left(v_{\ell}^{i}, v_{r}^{j}\right) \in E$ which is not covered. This implies in $G^{\prime}$ the existence of a path from $x_{i}$ (or $x_{i}^{\prime}$ ) to $x_{j}$, with $i<j$, passing through the gadget corresponding to the edge $\left(v_{\ell, 2}^{i}, v_{r, 1}^{j}\right)$. Hence,

- if $i=1$ and $j=2$ then $\operatorname{opt}\left(I^{\prime}\right) \leq 3$

- if $i=1$ and $j=3$ then $\operatorname{opt}\left(I^{\prime}\right) \leq 3$ 
- if $i=2$ and $j=3$ then $\operatorname{opt}\left(I^{\prime}\right) \leq 3$

Therefore, $\operatorname{opt}\left(I^{\prime}\right) \leq 3$.

Theorem 2. $k$ Most Vital Nodes 1-Median and $k$ Most Vital Nodes 1-CENTER are NP-hard to approximate within a factor $\frac{3}{2}-\epsilon$, for any $\epsilon>0$.

Proof: We first consider $k$ Most Vital Nodes 1-Median.

Let $I$ be an instance of MiN VerTex Cover formed by a graph $G=(V, E)$ with a tripartition $V=V_{1} \cup V_{2} \cup V_{3}$ and $|V|=n$. We construct an instance $I^{\prime}$ of $k$ Most Vital Nodes 1-Median consisting of a graph $G^{\prime}=\left(V^{\prime}, E^{\prime}\right)$ with $k<n$ as follows (see Figure 3). $G^{\prime}$ is a copy of $G$ to which we add complete graphs $K_{n}^{i}$ with $n$ nodes $x_{i}^{1}, \ldots, x_{i}^{n}$ for $i=1,2,3$. We connect each node $v_{\ell}^{i} \in V_{i}$ with each node $x_{i}^{r}$, for $i=1,2,3, \ell=1, \ldots,\left|V_{i}\right|$ and $r=1, \ldots, n$. We connect also each node $x_{i}^{r}$ to each node $x_{i+1}^{r}$ for $i=1,2$ and $r=1, \ldots, n$. We assign a distance 2 to edges $\left(x_{i}^{r}, x_{i+1}^{r}\right)$ for $i=1,2$ and $r=1, \ldots, n$, a distance 1 to edges $\left(x_{1}^{r}, v_{\ell}^{1}\right)$ for $\ell=1, \ldots,\left|V_{1}\right|$ and $r=1, \ldots, n$ and a distance 0 to all other edges in $E^{\prime}$. We set $w_{x_{1}^{r}}=7$ and $w_{x_{2}^{r}}=w_{x_{3}^{r}}=1$ for $r=1, \ldots, n$, and $w_{v_{\ell}^{i}}=0$ for $i=1,2,3, \ell=1, \ldots,\left|V_{i}\right|$. We show in the following that:

1. opt $(I) \leq k \Rightarrow \operatorname{opt}\left(I^{\prime}\right) \geq 6 n$

2. $\operatorname{opt}(I)>k \Rightarrow \operatorname{opt}\left(I^{\prime}\right) \leq 4 n$

which proves that $k$ Most Vital Nodes 1-median is $N P$-hard to approximate within a factor $\frac{3}{2}-\epsilon$, for any $\epsilon>0$.

First observe that there exists at least one optimal solution of $k$ Most VitaL NODES 1-MEDIAN containing only nodes of $V$. Indeed, if a solution contains nodes from $K_{n}^{i}$ for some $i$, it must contain all nodes of $K_{n}^{i}$ in order to have a chance to increase the solution value by disconnecting these nodes from the graph. Therefore, since $k<n$, it is at least as good to select $k$ nodes in $V$ only.

Observe also that $G^{\prime}$ is designed so as to ensure that any node $x_{1}^{r}$ for $r=$ $1, \ldots, n$ will always be an optimal 1 -median node. Indeed, since the weight of a vertex $x_{1}^{r}$ is 7 and all edges incident to $x_{1}^{r}$, except the edges $\left(x_{1}^{r}, x_{1}^{j}\right)$ for $j=$ $1, \ldots, n$ and $j \neq r$ have distance at least 1 , any other node would have a total weighted distance of at least 7 , while any node $x_{1}^{r}$ has always a total weighted distance of at most 6 . We consider arbitrarily in the following that $x_{1}^{1}$ is the selected optimal 1-median node.

1. If there exists a vertex cover $V^{\prime} \subseteq V$ of cardinality less than $k$ in $G$ then consider any set of vertices $V^{\prime \prime} \supset V^{\prime}$ of cardinality $k$, and remove $V^{\prime \prime}$ from $G^{\prime}$. Taking $x_{1}^{1}$ as the optimal 1-median node in $G^{\prime}-V^{\prime \prime}$, we get a total weighted distance $\sum_{j=1}^{n}\left(d\left(x_{1}^{1}, x_{2}^{j}\right)+d\left(x_{1}^{1}, x_{3}^{j}\right)\right)=\sum_{j=1}^{n}(2+4)=6 n$. Hence, $\operatorname{opt}\left(I^{\prime}\right) \geq 6 n$.

2. Let $N^{*} \subseteq V$ be any solution of $k$ Most Vital Nodes 1-MEdian which contains only nodes of $V$. Taking $x_{1}^{1}$ as the optimal 1-median node in $G^{\prime}-N^{*}$, we get $\operatorname{opt}\left(I^{\prime}\right)=\sum_{\ell=1}^{n}\left(d\left(x_{1}^{1}, x_{2}^{\ell}\right)+d\left(x_{1}^{1}, x_{3}^{\ell}\right)\right)$. Since $\left|N^{*}\right|=k$ and $\operatorname{opt}(I)>k$, 

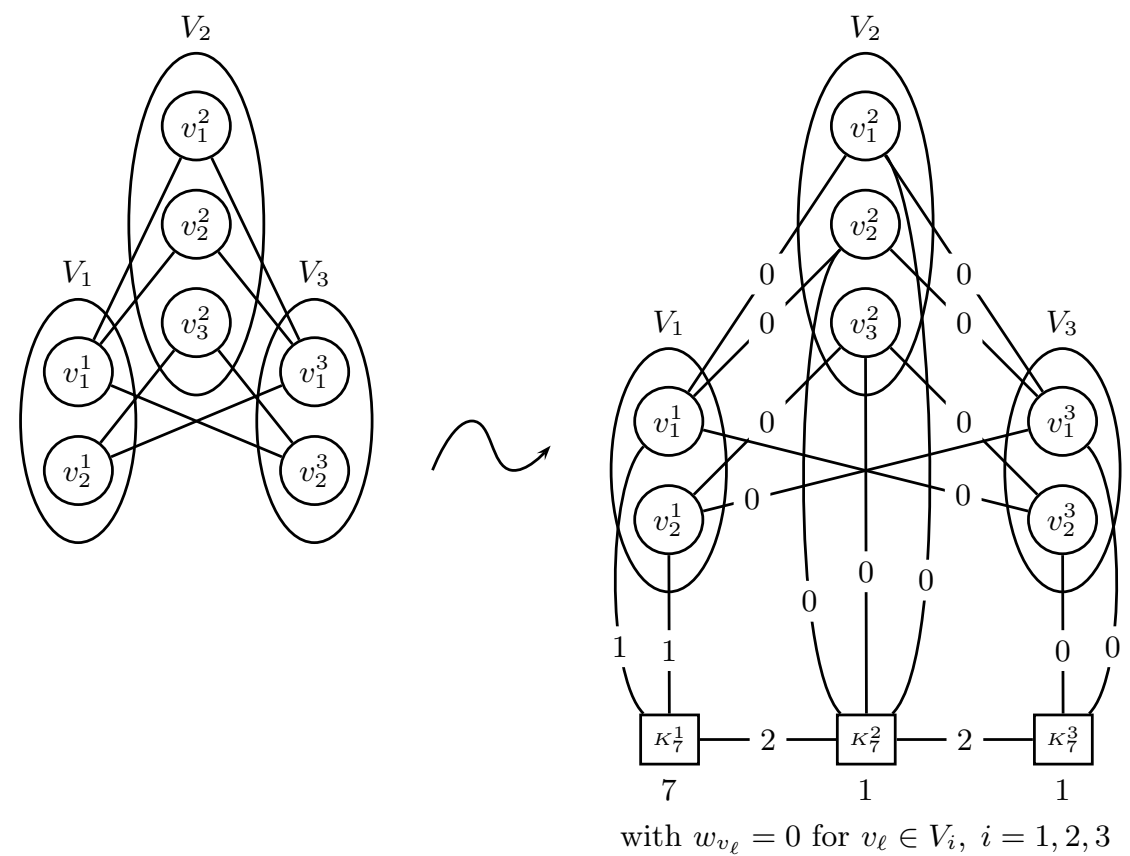

Fig. 3. Construction of $G^{\prime}$ from $G$

$N^{*}$ is not a vertex cover in $G$. Thus, there exists at least one edge $\left(v_{i}, v_{j}\right) \in E$ which is not covered. This implies in $G^{\prime}$ the existence of a path from each $x_{i}^{r}$ to each $x_{j}^{r}$ for $r=1, \ldots, n$, passing through the edge $\left(v_{i}, v_{j}\right)$. Hence,

- if $i=1$ and $j=2$ then $\operatorname{opt}\left(I^{\prime}\right) \leq \sum_{\ell=1}^{n}(1+3)=4 n$

- if $i=1$ and $j=3$ then $\operatorname{opt}\left(I^{\prime}\right) \leq \sum_{\ell=1}^{n}(2+1)=3 n$

- if $i=2$ and $j=3$ then $\operatorname{opt}\left(I^{\prime}\right) \leq \sum_{\ell=1}^{n}(2+2)=4 n$

Consequently, opt $\left(I^{\prime}\right) \leq 4 n$.

We consider now $k$ Most Vital Nodes 1-Center. We use the same construction as above, but we modify the distance associated to the edges $\left(x_{2}^{r}, x_{3}^{r}\right)$ for $r=1, \ldots, n$ for which we assign a distance 1 . We show that:

1. $\operatorname{opt}(I) \leq k \Rightarrow \operatorname{opt}\left(I^{\prime}\right) \geq 3$

2. $\operatorname{opt}(I)>k \Rightarrow \operatorname{opt}\left(I^{\prime}\right) \leq 2$

which proves that $k$ Most Vital Nodes 1-CEnter is $N P$-hard to approximate within a factor $\frac{4}{3}-\epsilon$, for any $\epsilon>0$.

As previously, we can show that only the nodes of $V$ can be removed. We observe as above that any node $x_{1}^{r}$ for $r=1, \ldots, n$ will always be an optimal 1-center node. We consider arbitrarily in the following that $x_{1}^{1}$ is the selected optimal 1-center node. 
1. If there exists a vertex cover $V^{\prime} \subseteq V$ of cardinality less than $k$ in $G$ then consider any set of vertices $V^{\prime \prime} \supset V^{\prime}$ of cardinality $k$, and remove $V^{\prime \prime}$ from $G^{\prime}$. Taking $x_{1}^{1}$ as the optimal 1-center node in $G^{\prime}-V^{\prime \prime}$, we get a maximum weighted distance $\max \left\{\max _{j=1, \ldots, n} d\left(x_{1}^{1}, x_{2}^{j}\right), \max _{j=1, \ldots, n} d\left(x_{1}^{1}, x_{3}^{j}\right)\right\}=3$. Hence, opt $\left(I^{\prime}\right) \geq 3$.

2. Let $N^{*} \subseteq V$ be any solution of $k$ Most Vital Nodes 1-CEnTER which contains only nodes of $V$. Taking $x_{1}^{1}$ as the optimal 1-center node in $G^{\prime}-N^{*}$, we get $\operatorname{opt}\left(I^{\prime}\right)=\max \left\{\max _{\ell=1, \ldots, n} d\left(x_{1}^{1}, x_{2}^{\ell}\right), \max _{\ell=1, \ldots, n} d\left(x_{1}^{1}, x_{3}^{\ell}\right)\right\}$. Since $\left|N^{*}\right|=k$ and $\operatorname{opt}(I)>k, N^{*}$ is not a vertex cover in $G$. Thus, there exists at least one edge $\left(v_{i}, v_{j}\right) \in E$ which is not covered. This implies in $G^{\prime}$ the existence of a path from each $x_{i}^{r}$ to each $x_{j}^{r}$ for $r=1, \ldots, n$, passing through the edge $\left(v_{i}, v_{j}\right)$. Hence,

- if $i=1$ and $j=2$ then $\operatorname{opt}\left(I^{\prime}\right)=\max \left\{d\left(x_{1}^{1}, x_{2}^{1}\right), d\left(x_{1}^{1}, x_{3}^{1}\right)\right\} \leq 2$

- if $i=1$ and $j=3$ then $\operatorname{opt}\left(I^{\prime}\right)=d\left(x_{1}^{1}, x_{2}^{1}\right) \leq 2$

- if $i=2$ and $j=3$ then $\operatorname{opt}(I \prime)=\max \left\{d\left(x_{1}^{1}, x_{2}^{1}\right), d\left(x_{1}^{1}, x_{3}^{1}\right)\right\} \leq 2$.

Therefore, $\operatorname{opt}\left(I^{\prime}\right) \leq 2$.

We prove now that the four problems Min Edge (Node) BlOCKER 1MEdiAn and Min Edge (NODE) BLOCKER 1-CENTER are not 1.36 approximable, unless $P=N P$. These results, stated in theorems 3 and 4 , are obtained by constructing $E$-reductions from Min VerTex Cover shown $N P$-hard to approximate within a factor 1.36 [5].

Theorem 3. Min Edge Blocker 1-median and Min Edge Blocker 1CENTER are NP-hard to approximate within a factor 1.36 .

Proof: We first consider Min Edge BLOcker 1-MEdian.

Let $I$ be an instance of Min VerTex Cover consisting of a graph $G=(V, E)$ with $V=\left\{v_{1}, \ldots, v_{n}\right\}$. We construct an instance $I^{\prime}$ of Min Edge BLOCKER 1MEDIAN formed by a graph $G^{\prime}=\left(V^{\prime}, E^{\prime}\right)$ and a positive integer $U$ as follows (see Figure 4). We associate for each node $v_{i} \in V$ two nodes $v_{i}$ and $v_{i}^{\prime}$ in $V^{\prime}$ and connect them in $E^{\prime}$ for $i=1, \ldots, n$. We add for each edge $\left(v_{i}, v_{j}\right) \in E$, with $i<j$, an edge $\left(v_{i}^{\prime}, v_{j}\right)$ to $E^{\prime}$. We also add $2 n$ nodes $x_{1}, x_{1}^{\prime}, x_{2}, x_{2}^{\prime}, \ldots, x_{n}, x_{n}^{\prime}$ connected by the path $\left(x_{1}, x_{1}^{\prime}\right),\left(x_{1}^{\prime}, x_{2}\right),\left(x_{2}, x_{2}^{\prime}\right),\left(x_{2}^{\prime}, x_{3}\right), \ldots,\left(x_{n-1}^{\prime}, x_{n}\right),\left(x_{n}, x_{n}^{\prime}\right)$. Finally, we connect $x_{i}$ to $v_{i}$ and $x_{i}^{\prime}$ to $v_{i}^{\prime}$ for $i=1, \ldots, n$. We assign the following distances to the edges of $E^{\prime}: d_{v_{i} v_{i}^{\prime}}=0, d_{x_{i} v_{i}}=d_{x_{i}^{\prime} v_{i}^{\prime}}=1$ and $d_{x_{i} x_{i}^{\prime}}=2$ for $i=1, \ldots, n$, $d_{x_{i}^{\prime} x_{i+1}}=0$ for $i=1, \ldots, n-1$ and $d_{v_{i}^{\prime} v_{j}}=2(j-i)-1$ for $\left(v_{i}, v_{j}\right) \in E$ and $i<j$. We set $w_{x_{1}}=2 n^{2}+1, w_{x_{i}}=1$ for $i=2, \ldots, n, w_{x_{i}^{\prime}}=1$ and $w_{v_{i}}=w_{v_{i}^{\prime}}=0$ for $i=1, \ldots, n$ and we consider that $U=2 n^{2}$. We replace each edge of $E^{\prime}$, except the edges $\left(v_{i}, v_{i}^{\prime}\right)$ for $i=1, \ldots, n$, by the gadget given in Figure 2 where each edge is replaced by $n+1$ instead of $n$ disjoint paths of length 2 (for edges $\left(x_{1}, v_{1}\right)$ and $\left(x_{1}, x_{1}^{\prime}\right), x_{1}$ plays the role of $i$ in Figure 2).

Observe that $G^{\prime}$ is designed so as to ensure that $x_{1}$ will always be the optimal 1-median node. Indeed, since the weight of vertex $x_{1}$ is $2 n^{2}+1$ and all 


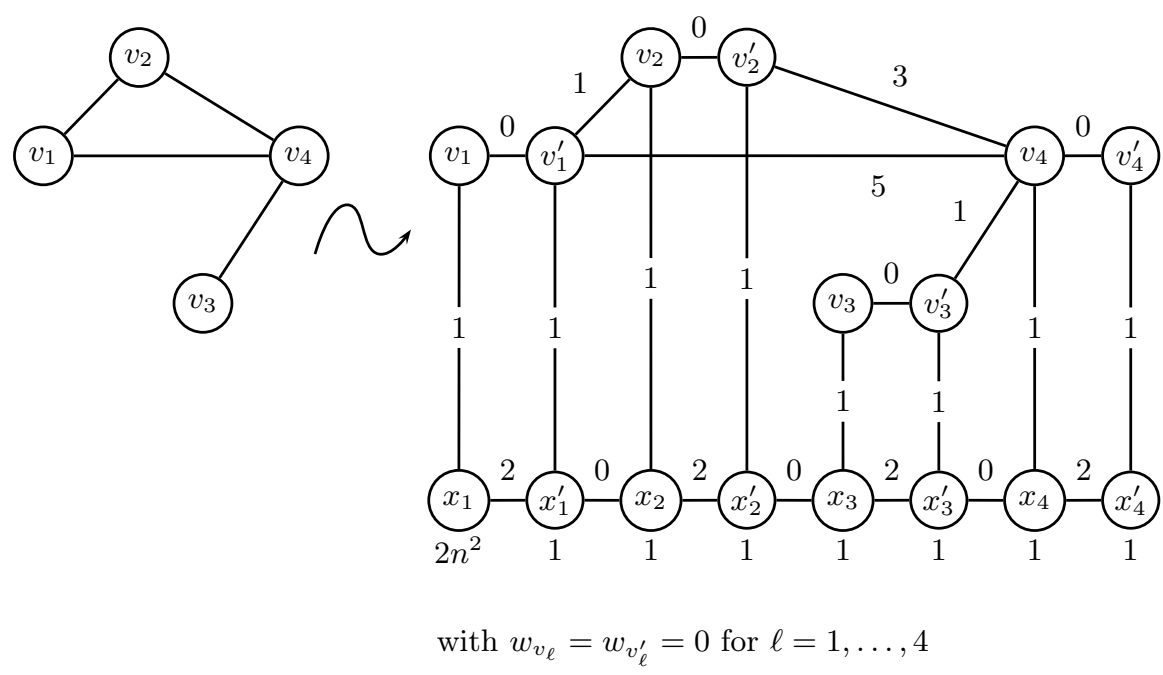

Fig. 4. Construction of $G^{\prime}$ from $G$ with $n=4$ nodes

edges incident to $x_{1}$ have distance at least 1 , any other node would have a total weighted distance of at least $2 n^{2}+1$. In the following, $x_{1}$ has always a total distance of at most $2 n^{2}$.

We prove first that $\operatorname{opt}\left(I^{\prime}\right) \leq \operatorname{opt}(I)$. Let $V^{*} \subseteq V$ be a minimum vertex cover of $G$. Let us consider $S^{*}=\left\{\left(v_{i}, v_{i}^{\prime}\right): v_{i} \in V^{*}\right\}$. By removing the edges in $S^{*}$ from $G^{\prime}$, the optimal 1-median node is $x_{1}$ with a total weighted distance $\sum_{i=1}^{n} w_{x_{i}} d\left(x_{1}, x_{i}\right)+\sum_{i=1}^{n} w_{x_{i}^{\prime}} d\left(x_{1}, x_{i}^{\prime}\right)=2\left(\sum_{i=1}^{n-1} i+\sum_{i=1}^{n} i\right)=2 n^{2}=U$. Hence, $\operatorname{opt}\left(I^{\prime}\right) \leq\left|S^{*}\right|=\operatorname{opt}(I)$.

When we remove all edges $\left(v_{i}, v_{i}^{\prime}\right)$, for $i=1, \ldots, n$ from $G^{\prime}$, the optimal 1-median node in the resulting graph is $x_{1}$ with value $U$. Hence, opt $\left(I^{\prime}\right) \leq n$. Let $S \subseteq E^{\prime}$ be an edge blocker for $G^{\prime}$. If $S$ contains an edge $\left(i, e_{i j}^{\ell}\right)$ or $\left(e_{i j}^{\ell}, j\right)$ from a gadget corresponding to an initial edge $(i, j)$, it must contain at least $n+1$ edges from this gadget in order to suppress the communication between $i$ and $j$, otherwise the value of an optimal solution for the 1-median problem in $G^{\prime}-S$ is the same as in $G^{\prime}-\left(S \backslash\left\{\left(i, e_{i j}^{\ell}\right)\right\}\right)$ or $G^{\prime}-\left(S \backslash\left\{\left(e_{i j}^{\ell}, j\right)\right\}\right)$. Therefore, since opt $\left(I^{\prime}\right) \leq n$, we can consider in the following that $S$ contains only edges among the edges $\left(v_{i}, v_{i}^{\prime}\right)$, $i \in\{1, \ldots, n\}$.

Let us consider $N=\left\{v_{i}:\left(v_{i}, v_{i}^{\prime}\right) \in S\right\}$ where $S$ is an edge blocker. We prove, by contradiction, that $N$ is a vertex cover in $G$. Suppose that there exists an edge $\left(v_{i}, v_{j}\right) \in E$ such that $v_{i} \notin N, v_{j} \notin N$ and $i<j$. We show in the following that by removing $S$ from $G^{\prime}$, the value of an optimal solution for the 1 -median problem in the remaining graph is strictly less than $2 n^{2}$. Indeed, $x_{1}$ is the optimal 1-median node in $G^{\prime}-S$. Let $D\left(x_{1}\right)$ be the total weighted distance 
associated to $x_{1}$ in $G^{\prime}-S$. We have $D\left(x_{1}\right)=\sum_{\ell=1}^{n} d\left(x_{1}, x_{\ell}^{\prime}\right)+\sum_{\ell=1}^{n} d\left(x_{1}, x_{\ell}\right)=$ $\sum_{\ell=1}^{j-1} d\left(x_{1}, x_{\ell}^{\prime}\right)+d\left(x_{1}, x_{j}^{\prime}\right)+\sum_{\ell=j+1}^{n} d\left(x_{1}, x_{\ell}^{\prime}\right)+\sum_{\ell=1}^{n} d\left(x_{1}, x_{\ell}\right)$. Then, $D\left(x_{1}\right) \leq$ $2 \sum_{\ell=1}^{j-1} \ell+d\left(x_{1}, x_{j}^{\prime}\right)+2 \sum_{\ell=j+1}^{n} \ell+2 \sum_{\ell=1}^{n-1} \ell=2 \sum_{\ell=1}^{n} \ell-2 j+d\left(x_{1}, x_{j}^{\prime}\right)+$ $2 \sum_{\ell=1}^{n-1} \ell=2 n^{2}-2 j+d\left(x_{1}, x_{j}^{\prime}\right)$. The edge $\left(v_{i}, v_{j}\right)$ being not covered, this implies the existence of a path from $x_{1}$ to $x_{j}^{\prime}$ using a subpath from $x_{1}$ to $x_{i}$ and joining $x_{i}$ to $x_{j}^{\prime}$ by a subpath passing through the gadget associated to the edge $\left(v_{i}, v_{j}\right)$. We have $d\left(x_{1}, x_{j}^{\prime}\right) \leq 2(i-1)+1+2(j-i)-1+1=2 j-1$. Thus, we have $D\left(x_{1}\right) \leq 2 n^{2}-1<2 n^{2}$, contradicting the assumption that $S$ is an edge blocker. Therefore, $N$ is a vertex cover in $G$ such that $\operatorname{val}(I, N)=\operatorname{val}\left(I^{\prime}, S\right)$. Consequently, $\varepsilon(I, N)=\frac{\operatorname{val}(I, N)}{\operatorname{opt}(I)}-1 \leq \frac{\operatorname{val}\left(I^{\prime}, S\right)}{\operatorname{opt}\left(I^{\prime}\right)}-1=\varepsilon\left(I^{\prime}, S\right)$, which achieves the proof.

We consider now Min EDGE BLOCKER 1-CENTER.

We use the same construction as above with $U=2 n$. As above, $G^{\prime}$ is designed so as to ensure that $x_{1}$ will always be the optimal 1-center node.

We show first that $\operatorname{opt}\left(I^{\prime}\right) \leq \operatorname{opt}(I)$. Let $V^{*} \subseteq V$ be a minimum vertex cover in $G$. Let us consider $S^{*}=\left\{\left(v_{i}, v_{i}^{\prime}\right): v_{i} \in V^{*}\right\}$. By removing the edges of $S^{*}$ from the graph $G^{\prime}$, the optimal 1-center node is $x_{1}$ with a maximum weighted distance $d\left(x_{1}, x_{n}^{\prime}\right)=2 n=U$. Hence, opt $\left(I^{\prime}\right) \leq\left|S^{*}\right|=\operatorname{opt}(I)$.

Let $S \subseteq E^{\prime}$ be an edge blocker. We can assume, similarly to the 1-median problem, that $S$ contains only edges among the edges $\left(v_{i}, v_{i}^{\prime}\right), i \in 1, \ldots, n$. Let us consider $N=\left\{v_{i}:\left(v_{i}, v_{i}^{\prime}\right) \in S\right\}$. In the following, we show by contradiction that $N$ is a vertex cover in $G$. Suppose that there exists an edge $\left(v_{i}, v_{j}\right) \in E$ such that $v_{i} \notin N, v_{j} \notin N$ and $i<j$. Then $x_{1}$ is the optimal 1-center node in $G^{\prime}-S$ with a maximum weighted distance $D_{\max }\left(x_{1}\right)=d\left(x_{1}, x_{n}\right)$. The edge $\left(v_{i}, v_{j}\right)$ being not covered, this implies the existence of a path from $x_{1}$ to $x_{j}^{\prime}$ using a subpath from $x_{1}$ to $x_{i}$ and joining $x_{i}$ to $x_{j}^{\prime}$ by a subpath passing through the gadget associated to the edge $\left(v_{i}, v_{j}\right)$. Then $D_{\max }\left(x_{1}\right) \leq 2(i-1)+1+2(j-i)-$ $1+1+2(n-j)=2 n-1<2 n$, contradicting the assumption that $S$ is an edge blocker. Therefore $N$ is a vertex cover in $G$ such that $\operatorname{val}(I, N)=\operatorname{val}\left(I^{\prime}, S\right)$. Consequently, $\varepsilon(I, N)=\frac{\operatorname{val}(I, N)}{\operatorname{opt}(I)}-1 \leq \frac{\operatorname{val}\left(I^{\prime}, S\right)}{\operatorname{opt}\left(I^{\prime}\right)}-1=\varepsilon\left(I^{\prime}, S\right)$, which achieves the proof.

Theorem 4. Min Node Blocker 1-Median and Min Node BLOCKer 1CENTER are NP-hard to approximate within a factor 1.36.

Proof: We consider first Min Node BLOCKER 1-Median.

Let $I$ be an instance of MiN VERTEX COVER consisting of a graph $G=(V, E)$ with $V=\left\{v_{1}, \ldots, v_{n}\right\}$. We construct an instance $I^{\prime}$ of Min NoDE BLOCKER 1MEDIAN formed by a graph $G^{\prime}=\left(V^{\prime}, E^{\prime}\right)$ and a positive integer $U$ as follows (see Figure 5). $G^{\prime}$ is a copy of $G$ to which we add one node $x_{1}$ and complete graphs $K_{n+1}^{i}$ with $n+1$ nodes $x_{i}^{1}, \ldots, x_{i}^{n+1}$ for $i=2, \ldots, n$. We connect $x_{1}$ to $v_{1}$ and $x_{2}^{r}$ for $r=1, \ldots, n+1$, and each node $x_{i}^{r}$ to $v_{i}$ for $i=2, \ldots, n$ and $r=1, \ldots, n+1$. We also connect each node $x_{i}^{r}$ to each node $x_{i+1}^{r}$ for 
$r=1, \ldots, n+1$ and $i=2, \ldots, n-1$. We assign a distance 1 to the edge $\left(x_{1}, v_{1}\right)$, a distance 2 to the edges $\left(x_{1}, x_{2}^{r}\right)$ and $\left(x_{i}^{r}, x_{i+1}^{r}\right)$ for $i=2, \ldots, n-1$ and $r=1, \ldots, n+1$, and a distance 0 to all other edges in $E^{\prime}$. Let us set $w_{x_{1}}=n^{3}$, $w_{x_{i}^{r}}=1$ for $i=2, \ldots, n$ and $r=1, \ldots, n+1$ and $w_{v_{i}}=0$ for $i=1, \ldots, n$. Finally, we set $U=n\left(n^{2}-1\right)$.
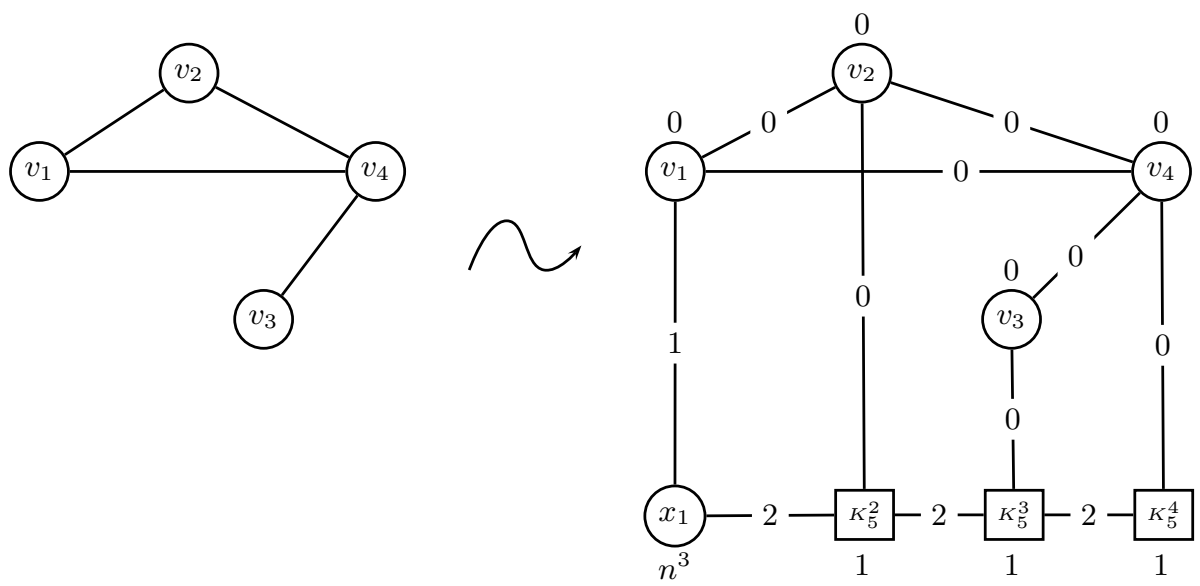

Fig. 5. Construction of $G^{\prime}$ from $G$ with $n=4$ nodes

Observe that $G^{\prime}$ is designed so as to ensure that $x_{1}$ will always be the optimal 1-median node. Indeed, since the weight of vertex $x_{1}$ is $n^{3}$ and all edges incident to $x_{1}$ have distance at least 1 , any other node would have a total weighted distance of at least $n^{3}$. In the following, $x_{1}$ has always a total distance of at most $n\left(n^{2}-1\right)$.

We show first that $\operatorname{opt}\left(I^{\prime}\right) \leq \operatorname{opt}(I)$. Let $V^{*} \subseteq V$ be a minimum vertex cover in $G$. By removing $V^{*}$ from $G^{\prime}$, the optimal 1 -median node is $x_{1}$ with a total weighted distance $\sum_{\ell=2}^{n} \sum_{r=1}^{n+1} d\left(x_{1}, x_{\ell}^{r}\right)=2(n+1) \sum_{i=1}^{n-1} i=n(n-1)(n+1)=U$. Hence, $\operatorname{opt}\left(I^{\prime}\right) \leq\left|V^{*}\right|=\operatorname{opt}(I)$.

Let $N \subseteq V^{\prime}$ be a node blocker. According to the construction of $G^{\prime}$, in order to obtain an optimal solution for the 1-median problem in the graph $G^{\prime}-N$ of a value at least $U, N$ must be included in $V$. We show, by contradiction, that $N$ is a vertex cover in $G$. Suppose that there exists an edge $\left(v_{i}, v_{j}\right) \in E$ such that $v_{i} \notin N, v_{j} \notin N$ and $i<j$. The optimal 1-median node in $G^{\prime}-N$ is $x_{1}$ with value strictly less than $n(n-1)(n+1)$. Indeed, let $D\left(x_{1}\right)$ be the total weighted distance associated to $x_{1}$ in $G^{\prime}-N$. Hence, $D\left(x_{1}\right)=\sum_{\ell=2}^{n} \sum_{r=1}^{n+1} d\left(x_{1}, x_{\ell}^{r}\right)=$ $\left.\sum_{\ell=2}^{j-1} \sum_{r=1}^{n+1} d\left(x_{1}, x_{\ell}^{r}\right)+\sum_{r=1}^{n+1} d\left(x_{1}, x_{j}^{r}\right)+\sum_{r=1}^{n+1} \sum_{\ell=j+1}^{n} d\left(x_{1}, x_{\ell}^{r}\right)\right)$. We distinguish two cases: 
- If $v_{i}=v_{1}$ then $d\left(x_{1}, x_{j}^{r}\right)=d_{x_{1} v_{1}}+d_{v_{1} v_{j}}+d_{v_{j} x_{j}^{r}}=1$ for $r=1, \ldots, n+1$. Hence, we obtain $D\left(x_{1}\right) \leq 2(n+1) \sum_{\ell=1}^{j-2} \ell+(n+1)+2(n+1) \sum_{\ell=j}^{n-1} \ell<$ $2(n+1) \sum_{\ell=1}^{j-2} \ell+2(j-1)(n+1)+2(n+1) \sum_{\ell=j}^{n-1} \ell=n(n-1)(n+1)$, contradiction.

- If $v_{i} \neq v_{1}$ then $d\left(x_{1}, x_{j}^{r}\right)=d\left(x_{1}, x_{i}^{1}\right)+d_{x_{i}^{1} v_{i}}+d_{v_{i} v_{j}}+d_{v_{j} x_{j}^{r}}=d\left(x_{1}, x_{i}^{1}\right)$ for $r=1, \ldots, n+1$. Hence, we obtain $D\left(x_{1}\right) \leq 2(n+1) \sum_{\ell=1}^{j-2} \ell+2(i-1)(n+$ 1) $+2(n+1) \sum_{\ell=j}^{n-1} \ell<2(n+1) \sum_{\ell=1}^{j-2} \ell+2(j-1)(n+1)+2(n+1) \sum_{\ell=j}^{n-1} \ell=$ $n(n-1)(n+1)$, contradiction.

Therefore $N$ is a vertex cover in $G$ such that $\operatorname{val}(I, N)=\operatorname{val}\left(I^{\prime}, N\right)$. Consequently, $\varepsilon(I, N)=\frac{\operatorname{val}(I, N)}{\operatorname{opt}(I)}-1 \leq \frac{\operatorname{val}\left(I^{\prime}, N\right)}{\operatorname{opt}\left(I^{\prime}\right)}-1=\varepsilon\left(I^{\prime}, N\right)$, which achieves the proof.

We consider now Min Node BLOCKER 1-CENTER.

We use the same construction as above with $U=2(n-1)$. Here again, we observe that $G^{\prime}$ is designed so as to ensure that $x_{1}$ will always be the optimal 1-center node.

We show first that $\operatorname{opt}\left(I^{\prime}\right) \leq \operatorname{opt}(I)$. Let $V^{*} \subseteq V$ be a minimum vertex cover in $G$. By deleting the nodes of $V^{*}$ from $G^{\prime}$, the optimal 1-center node in the remaining graph is $x_{1}$ with a maximum weighted distance $d\left(x_{1}, x_{n}^{r}\right)=$ $2(n-1)=U$ for any $r=1, \ldots, n+1$. Hence, $\operatorname{opt}\left(I^{\prime}\right) \leq\left|V^{*}\right|=\operatorname{opt}(I)$.

When we remove all nodes $v_{i}, i=1, \ldots, n$ from $G^{\prime}$, the optimal 1-center node in the resulting graph is $x_{1}$ with value $U$. Hence, opt $\left(I^{\prime}\right) \leq n$. Let $N \subseteq V^{\prime}$ be a node blocker. According to the construction of $G^{\prime}$, in order to obtain an optimal 1-center node in $G^{\prime}-N$ of value at least $U, N$ cannot contain $x_{1}$. If $N$ contains nodes $x_{i}^{\ell}$ for a given $i$ and $\ell$, then $N$ must contains all the $n+1$ nodes $x_{i}^{r}$ for $r=1, \ldots, n+1$, otherwise the value of an optimal solution for the 1-center problem in $G^{\prime}-N$ is the same as in $G^{\prime}-\left(N \backslash\left\{x_{i}^{\ell}\right\}\right)$. Therefore, since $\operatorname{opt}\left(I^{\prime}\right) \leq n$, we can consider in the following that $N$ is included in $V$. In the following, we prove by contradiction that $N$ forms a vertex cover in $G$. Suppose that there exists an edge $\left(v_{i}, v_{j}\right) \in E$ such that $v_{i} \notin N, v_{j} \notin N$ and $i<j$. By removing $N$ from $G^{\prime}$, the optimal 1-center node is $x_{1}$ with a maximum weighted distance $D_{\max }\left(x_{1}\right)=d\left(x_{1}, x_{n}^{r}\right)$ for any $r=1, \ldots, n$. We distinguish two cases:

- if $v_{i}=v_{1}$ then $D_{\max }\left(x_{1}\right)=d_{x_{1} v_{1}}+d_{v_{1} v_{j}}+d_{v_{j} x_{j}^{1}}+d\left(x_{j}^{1}, x_{n}^{r}\right) \leq 1+0+0+$ $2(n-j) \leq 1+2 n-4<2(n-1)$, contradiction.

- if $v_{i} \neq v_{1}$ then $D_{\max }\left(x_{1}\right) \leq d\left(x_{1}, x_{i}^{1}\right)+d_{x_{i}^{1} v_{i}}+d_{v_{i} v_{j}}+d_{v_{j} x_{j}^{1}}+d\left(x_{j}^{1}, x_{n}^{r}\right) \leq$ $2(i-1)+0+0+0+2(n-j)=2(n-1)-2(j-i)<2(n-1)$, contradiction.

Therefore $N$ is a vertex cover in $G$ such that $\operatorname{val}(I, N)=\operatorname{val}\left(I^{\prime}, N\right)$. Consequently, $\varepsilon(I, N)=\frac{\operatorname{val}(I, N)}{\operatorname{opt}(I)}-1 \leq \frac{\operatorname{val}\left(I^{\prime}, N\right)}{\operatorname{opt}\left(I^{\prime}\right)}-1=\varepsilon\left(I^{\prime}, N\right)$, which achieves the proof. 


\section{Conclusion}

We established in this paper negative results concerning the approximation of $k$ most vital edges (nodes) and min edge (node) blocker versions of the 1-median and 1-center location problems. An interesting open question would be to establish positive results concerning the approximability of these problems. Another interesting perspective is to find efficient exact algorithms to solve them.

\section{References}

1. S. Arora and C. Lund. Hardness of approximations. In Approximation algorithms for NP-hard problems, pages 399-446. PWS Publishing Company, 1996.

2. A. Bar-Noy, S. Khuller, and B. Schieber. The complexity of finding most vital arcs and nodes. Technical Report CS-TR-3539, Department of Computer Science, University of Maryland, 1995.

3. C. Bazgan, S. Toubaline, and Zs. Tuza. Complexity of most vital nodes for independent set in graphs related to tree structures. In Proceedings of the $21^{\text {st }}$ International Workshop on Combinatorial Algorithms (IWOCA 2010). Springer, 2010. to appear in LNCS.

4. R. L. Brooks. On colouring the nodes of a network. Mathematical Proceeing of the Cambridge Philosophical Society, 37(2):194-197, 1941.

5. I. Dinur and S. Safra. On the hardness of approximating minimum vertex cover. Annals of Mathematics, 162(1):439-485, 2005.

6. G. N. Frederickson and R. Solis-Oba. Increasing the weight of minimum spanning trees. Proceedings of the 7th Annual ACM-SIAM Symposium on Discrete Algorithms (SODA 1996), pages 539-546, 1996.

7. M. R. Garey, D. S. Johnson, and L. Stockmeyer. Some simplified NP-complete graph problems. Theoretical Computer Science, 1(3):237-267, 1976.

8. L. Khachiyan, E. Boros, K. Borys, K. Elbassioni, V. Gurvich, G. Rudolf, and J. Zhao. On short paths interdiction problems : total and node-wise limited interdiction. Theory of Computing Systems, 43(2):204-233, 2008.

9. S. Khanna, R. Motwani, M. Sudan, and U. Vazirani. On syntactic versus computational views of approximability. In Proceedings of the 35th Annual IEEE Annual Symposium on Foundations of Computer Science (FOCS 1994), pages 819-830, 1994. Also published in SIAM Journal on Computing, 28(1), 1999, 164-191.

10. B. Ries, C. Bentz, C. Picouleau, D. de Werra, M. Costa, and R. Zenklusen. Blockers and transversals in some subclasses of bipartite graphs: When caterpillars are dancing on a grid. Discrete Mathematics, 310(1):132-146, 2010.

11. R. K. Wood. Deterministic network interdiction. Mathematical and Computer Modeling, 17(2):1-18, 1993.

12. R. Zenklusen, B. Ries, C. Picouleau, D. de Werra, M. Costa, and C. Bentz. Blockers and transversals. Discrete Mathematics, 309(13):4306-4314, 2009. 